Japan. J. Math.

Vol. 7, No. 1, 1981

\title{
On isoparametric hypersurfaces in the Lorentzian space forms*
}

\author{
By Katsumi Nomizu
}

(Received September 17, 1980)

The purpose of the present paper is to extend the basic identity concerning constant principal curvatures of an isoparametric hypersurface in E. Cartan's theory (see [1] and [2]) to the case of a spacelike hypersurface in any one of the standard Lorentzian space forms $M^{n+1}(c)$ of constant sectional curvature $c$.

A connected space-like hypersurface $M^{n}$ in $M^{n+1}(c)$ is said to be isoparametric if its shape operator $A$ has constant eigenvalues (principal curvatures). If $a_{1}, \cdots, a_{p}$ are all the distinct constant principal curvatures with multiplicities $\nu_{1}, \cdots, \nu_{p}$, respectively then we prove

Theorem. Let $p \geq 2$. For each $i, 1 \leq i \leq p$, we have

$$
\sum_{j \neq i} \nu_{j} \frac{-c+a_{i} a_{j}}{a_{i}-a_{j}}=0 .
$$

We must note that this identity differs from the classical case in that the sectional curvature $c$ of the ambient space appears with the minus sign in front. We present here a proof based on the consideration of focal varieties which works also in the classical case as mentioned in [2].

The identity above serves as the starting point for the study of isoparametric hypersurfaces in the Lorentzian space forms. As E. Cartan showed, the identity implies that $p \leq 2$ if $-c \leq 0$, that is, if $c \geq 0$. Thus in $L^{n+1}$ and $S_{1}^{n+1}(c), c>0$, an isoparametric hypersurface has at most two distinct constant principal curvatures. It is in $H_{1}^{n+1}(c), c<0$, that we can hope to find an isoparametric hypersurface with more than two distinct constant principal curvatures.

\section{§1. Geodesics in Lorentzian space forms}

We summarize the known facts about the Lorentzian space forms we

* Work partially supported by NSF Grant MCS79-01310. 
shall be working with. Let $R_{s}^{m}$ be the $m$-dimensional real vector space $R^{m}$ with an inner product of signature $(s, m-s)$ given by

$$
\langle x, y\rangle=-\sum_{i=1}^{s} x_{i} y_{i}+\sum_{j=s+1}^{m} x_{j} y_{j}
$$

for $x=\left(x_{1}, \cdots, x_{m}\right)$ and $y=\left(y_{1}, \cdots, y_{m}\right)$.

The flat Lorentz space $L^{n+1}$ is $R_{1}^{n+1}$ regarded as an affine space with parallel Lorentzian inner product $\langle$,$\rangle . The sectional curvature is 0$ and the geodesics are straight lines.

For a given positive number $c$, the standard space form $S_{1}^{n}(c)$ is the hypersurface

$$
\left\{x \in L^{n+1} ;\langle x, x\rangle=r^{2}\right\}, \quad r=\frac{1}{\sqrt{c}}
$$

with the induced Lorentzian metric. It has constant sectional curvature $c$.

Let $x_{0} \in S_{1}^{n}(c)$ and let $X_{0}$ be a tangent vector at $x_{0}$ (so that $\left\langle x_{0}, X_{0}\right\rangle=0$ ).

If $\left\langle X_{0}, X_{0}\right\rangle=1$, then the (space-like) geodesic with initial point $x_{0}$ and initial tangent vector $X_{0}$ is given by

$$
x_{t}=\cos \left(\frac{t}{r}\right) x_{0}+\sin \left(\frac{t}{r}\right) r X_{0}
$$

where $t$ is the affine parameter (arc length) of the geodesic.

If $\left\langle X_{0}, X_{0}\right\rangle=-1$, then the (time-like) geodesic with initial point $x_{0}$ and initial tangent vector $X_{0}$ is given by

$$
x_{t}=\cosh \left(\frac{t}{r}\right) x_{0}+\sinh \left(\frac{t}{r}\right) r X_{0},
$$

where $t$ is the affine parameter (proper time) of the geodesic.

If $\left\langle X_{0}, X_{0}\right\rangle=0$, then the (null) geodesic with initial point $x_{0}$ and initial tangent vector $X_{0}$ is given by

$$
x_{t}=x_{0}+t X_{0}
$$

where $t$ is an affine parameter.

For a given negative number $c$, the standard space form $H_{1}^{n}(c)$ is the hypersurface

$$
\left\{x \in R_{2}^{n+1} ;\langle x, x\rangle=-r^{2}\right\}, \quad r=\frac{1}{\sqrt{-c}},
$$

with the induced Lorentzian metric. It has constant sectional curvature $c$.

Let $x_{0} \in H_{1}^{n}(c)$ and let $X_{0}$ be a tangent vector at $x_{0}$. 
If $\left\langle X_{0}, X_{0}\right\rangle=1$, then the (space-like) geodesic with initial conditions $\left(x_{0}\right.$, $\left.X_{0}\right)$ is given by

$$
x_{t}=\cosh \left(\frac{t}{r}\right) x_{0}+\sinh \left(\frac{t}{r}\right) r X_{0},
$$

where $t$ is the affine parameter (arc length).

If $\left\langle X_{0}, X_{0}\right\rangle=-1$, then the (time-like) geodesic with initial conditions $\left(x_{0}, X_{0}\right)$ is given by

$$
x_{t}=\cos \left(\frac{t}{r}\right) x_{0}+\sin \left(\frac{t}{r}\right) r X_{0},
$$

where $t$ is the affine parameter (proper time).

If $\left\langle X_{0}, X_{0}\right\rangle=0$, then the (null) geodesic with initial conditions $\left(x_{0}, X_{\mathfrak{v}}\right)$ is given by

$$
x_{t}=x_{0}+t X_{0},
$$

where $t$ is an affine parameter.

\section{§2. Focal varieties}

In a Lorentzian space form $M_{1}^{n+1}(c)$ we consider a connected space-like hypersurface $M^{n}$. For a field of time-like unit normals $\xi$, the corresponding shape operator $A$ of $M^{n}$ is given by

$$
\tilde{\nabla}_{X} \xi=-A X,
$$

where $X$ is a tangent vector to $M$ and $\tilde{V}$ denotes covariant differentiation in $M_{1}^{n+1}(c)$. We suppose that $M^{n}$ is isoparametric with constant principal curvatures $a_{1}, \cdots, a_{p}$ of multiplicities $\nu_{1}, \cdots, \nu_{p}$, respectively.

As in the classical case (see [1], [2]) we may define the focal varieties of $M$. (All the considerations here will be done locally.)

For each $x \in M$, we denote by $\operatorname{Exp}_{x} t \xi_{x}$ the time-like geodesic with initial conditions $\left(x, \xi_{x}\right)$.

If $c=0$, let $a_{i}$ be any non-zero constant principal curvature. We define $f_{i}: M \rightarrow L^{n+1}$ by

$$
f_{i}(x)=\operatorname{Exp}_{x} \frac{1}{a_{i}} \xi_{x}=x+\frac{1}{a_{i}} \xi_{x} .
$$

If $c>0$, let $a_{i}=(1 / r) \operatorname{coth}\left(t_{i} / r\right), r=1 / \sqrt{c}$, and define $f_{i}: M \rightarrow S_{1}^{n+1}(c)$ by 


$$
f_{i}(x)=\operatorname{Exp}_{x} t_{i} \xi_{i}=\cosh \left(\frac{t_{i}}{r}\right) x+\sinh \left(\frac{t_{i}}{r}\right) r \xi_{x} .
$$

If $c<0$, let $a_{i}=(1 / r) \cot \left(t_{i} / r\right), 0<t_{i} / r<\pi, r=1 / \sqrt{-c}$, and define $f_{i}: M \rightarrow$ $H_{1}^{n+1}(c)$ by

$$
f_{i}(x)=\operatorname{Exp}_{x} t_{i} \xi_{x}=\cos \left(\frac{t_{i}}{r}\right) x+\sin \left(\frac{t_{i}}{r}\right) r \xi_{x} .
$$

In each case, it turns out that the differential $\left(f_{i}\right)_{*}$ is 0 on $T_{i}(x)=$ $\left\{X \in T_{x}(M) ; A_{i} X=a_{i} X\right\}$ and injective on the subspace $\sum_{j \neq i} T_{j}(x)$ of $T_{x}(M)$.

We may therefore consider $V_{i}=f_{i}(M)$ as an $\left(n-\nu_{i}\right)$-dimensional submanifold of $M_{1}^{n+1}(c)$, which we call the focal variety of $M$. It will also turn out that the number

$$
\sum_{j \neq i} \nu_{j} \frac{-c+a_{i} a_{j}}{a_{i}-a_{j}}
$$

is equal to the trace of the shape operator for $V_{i}$ corresponding to the unit normal in the direction of the geodesic $\operatorname{Exp}_{x}\left(t \xi_{x}\right)$ as it comes into the point $f_{i}(x)$. Thus, if we can prove that the mean curvature vector of $V_{i}$ is 0 , that is, $V_{i}$ is a "maximal" submanifold of $M_{1}^{n+1}(c)$, then we have the basic identity. We shall give the detail of these arguments in the case $c<0$, because it is the most interesting case as we explained in the introduction.

\section{§3. Proof for $H_{1}^{n+1}(c)$}

Now let $M^{n}$ be a spacelike hypersurface in $H_{1}^{n+1}(c)$ with distinct constant principal curvatures $a_{1}, \cdots, a_{p}$ with multiplicities $\nu_{1}, \cdots, \nu_{p}$, respectively, where $p \geq 2$. We let

$$
a_{i}=\frac{1}{r} \cot \left(\frac{t_{i}}{r}\right), \quad r=\frac{1}{\sqrt{-c}}, \quad 0<\frac{t_{i}}{r}<\pi .
$$

Consider the mapping

$$
x \in M \rightarrow f_{i}(x)=\cos \left(\frac{t_{i}}{r}\right) x+\sin \left(\frac{t_{i}}{r}\right) r \xi_{.} \in V_{i}
$$

as in the preceding section.

The differential $\left(f_{i}\right)_{*}$ maps $X \in T_{i}(x)$ into

$$
\left(\cos \frac{t_{i}}{r}\right) X+\sin \left(\frac{t_{i}}{r}\right)(-r A X)=\left(\cos \frac{t_{i}}{r}\right) X+\left(\sin \frac{t_{i}}{r}\right)\left(\cot \frac{t_{i}}{r}\right) X=0 .
$$


If $X \in T_{j}(x)$ with $j \neq i$, then

$$
\begin{aligned}
\left(f_{i}\right)_{*}(X) & =\left(\cos \frac{t_{i}}{r}\right) X-\left(\sin \frac{t_{i}}{r}\right)\left(\cot \frac{t_{j}}{r}\right) X \\
& =\left(\cos \frac{t_{i}}{r}-\sin \frac{t_{i}}{r} \cot \frac{t_{j}}{r}\right) X
\end{aligned}
$$

which is a non-zero multiple of $X$. This shows $\left(f_{i}\right)_{*}$ is injective on $\sum_{j \neq i} T_{j}(x)$.

It follows that the tangent space $T_{p}\left(V_{i}\right)$ at $p=f_{i}(x)$ may be considered as $\left(f_{i}\right)_{*}\left(\sum_{j \neq i} T_{j}(x)\right)=\sum_{j \neq i} T_{j}(x)$ by virtue of (3).

The geodesic

$$
\operatorname{Exp}_{x} t \xi_{x}=\cos \left(\frac{t}{r}\right) x+\sin \left(\frac{t}{r}\right) r \xi_{x}
$$

has the tangent vector

$$
\xi_{x}^{\prime}=-\sin \left(\frac{t_{i}}{r}\right) \frac{x}{r}+\cos \left(\frac{t_{i}}{r}\right) \xi_{x}
$$

at the point $p=f_{i}(x)$.

Obviously $\xi_{x}^{\prime}$ is normal to $V_{i}$ at $p$.

If we translate any vector $Z \in T_{i}(x)$ to $p$ (in $R_{2}^{n+2}$ ) then it can be considered as a tangent vector at $p$ to $H_{1}^{n+1}$ which is normal to $V_{i}$, as well as to $\xi_{x}^{\prime}$. It follows that the normal space $N_{p}\left(V_{i}\right)$ of $V_{i}$ in $H_{1}^{n+1}$ at $p$, which has dimension $\nu_{0}+1$, is spanned by $\xi_{x}^{\prime}$ and all $Z \in T_{i}(x)$. We now compute the trace of the shape operator of $V_{i}$ for $B_{\xi_{x}^{\prime}}$ and $B_{Z}, Z \in T_{i}(x)$.

Denoting by $D$ the covariant differentiation in $R_{2}^{4}$, we have from (5)

$$
D_{X} \xi^{\prime}=-\sin \left(\frac{t_{i}}{r}\right) \frac{X}{r}+\cos \left(\frac{t_{i}}{r}\right) D_{X} \xi
$$

Since $D_{X} \xi=\tilde{V}_{X} \xi=-A X$, we get for $X \in T_{j}(x)$

$$
\begin{aligned}
D_{X} \xi^{\prime} & =-\sin \left(\frac{t_{i}}{r}\right) \frac{X}{r}-\cos \left(\frac{t_{i}}{r}\right) \frac{\cot \left(t_{j} / r\right) X}{r} \\
& =\left(-\sin \frac{t_{i}}{r}-\cos \frac{t_{i}}{r} \cot \frac{t_{j}}{r}\right) \frac{X}{r},
\end{aligned}
$$

which is going to be equal to $\left(f_{i}\right)_{*}\left(-B_{\hat{\xi}_{x}^{\prime}} X\right)$. By virtue of (3) and (1) we get

$$
\begin{aligned}
B_{\xi^{\prime} x} X & =\frac{\sin \left(t_{i} / r\right)+\cos \left(t_{i} / r\right) \cot \left(t_{j} / r\right)}{\cos \left(t_{i} / r\right)-\sin \left(t_{i} / r\right) \cot \left(t_{j} / r\right)} \frac{X}{r}=\frac{1+\cot \left(t_{i} / r\right) \cot \left(t_{j} / r\right)}{\cot \left(t_{i} / r\right)-\cot \left(t_{j} / r\right)} \frac{X}{r} \\
& =\frac{1+r a_{i} r a_{j}}{r a_{i}-r a_{j}} \frac{X}{r}=\frac{1 / r^{2}+a_{i} a_{j}}{a_{i}-a_{j}} X
\end{aligned}
$$


i.e.

$$
B_{\xi_{x}^{\prime}} X=\frac{-c+a_{i} a_{j}}{a_{i}-a_{j}} X \text { for } X \in T_{j}(x)
$$

Thus

$$
\operatorname{trace} B_{\xi_{x}^{\prime}}=\sum_{j \neq i} \nu_{j} \frac{-c+a_{i} a_{j}}{a_{i}-a_{j}}
$$

To compute trace $B_{Z}$ for $Z \in T_{i}(x)$ regarded as a normal vector to $V_{i}$, we may extend $Z$ to a vector field belonging to the distribution $T_{i}$ in a neighborhood of $x$ and set

$$
\left(f_{i}\right)_{*}\left(B_{Z} X\right)=-\tilde{V}_{X} Z, \quad X \in T_{j}(x)
$$

where $Z$ on the right-hand side is a field of normal vectors to $V_{i}$ in a neighborhood of $x$.

We use

LemMa. Let $Z$ be a vector field belonging to the distribution $T_{i}$. If $X \in T_{j}(x), j \neq i$, then $\nabla_{X} Z$ is normal to $T_{j}(x)$.

Proof of Lemma. We extend $X$ to a vector field belonging to the distribution $T_{j}$. From Codazzi's equation

$$
\left(\nabla_{X} A\right)(Z)=\left(\nabla_{Z} A\right)(X)
$$

we obtain

$$
\sum_{k=1}^{p}\left(a_{i}-a_{k}\right)\left(\nabla_{X} Z\right)_{k}=\sum_{k=1}^{p}\left(a_{j}-a_{k}\right)\left(\nabla_{Z} X\right)_{k},
$$

where ()$_{k}$ denotes the $T_{k}$-component. Taking the $j$-th component of each side, we get

$$
\left(a_{i}-a_{j}\right)\left(\nabla_{X} Z\right)_{j}=0
$$

Since $a_{i} \neq a_{j}$, we have $\left(\nabla_{X} Z\right)_{j}=0$. Thus $\nabla_{X} Z \perp T_{j}(x)$.

We now take $Z \in T_{i}(x)$ and extend it to a vector field belonging to the distribution $T_{i}$. For $X \in T_{j}(x)$, we have

$$
\tilde{\nabla}_{X} Z=\nabla_{X} Z
$$

which is normal to $T_{j}(x)$ by the lemma. Thus

$$
\left(f_{i}\right)^{*}\left(-B_{Z} X\right)=\sum_{k \neq i, j}\left(\nabla_{X} Z\right)_{k}
$$


Hence

$$
B_{Z} X=-\sum_{k \neq i, j} \frac{1}{\cos \left(t_{i} / r\right)-\sin \left(t_{i} / r\right) \cot \left(t_{k} / r\right)}\left(\nabla_{X} Z\right)_{k} \quad \text { for } X \in T_{j}(x)
$$

by virtue of (3).

Since

$$
\left\langle B_{Z} X, X\right\rangle=0
$$

we have

$$
\text { trace } B_{Z}=0 \text {. }
$$

Let $M_{i}$ be an integral manifold of the distribution $T_{i}$ through the point $x$. We define a differentiable mapping $g_{i}$ of $M_{i}$ into $N_{p}\left(V_{i}\right)$ as follows (this is an idea of Münzer as mentioned in [2]).

First we remark that $f_{i}$ maps $M_{i}$ into the single point $p$, because $\left(f_{i}\right)_{*}$ vanishes on $T_{i}(y)$ for every point $y$ as we saw before. For any $y \in M_{i}$, we define $g_{i}(y)$ to be the tangent vector at $p=f_{i}(y)$ of the geodesic $\operatorname{Exp}_{y} t \xi_{y}=$ $\cos (t / r) y+\sin (t / r) r \xi_{y}$. Thus

$$
g_{i}(y)=-\sin \frac{t_{i}}{r} \frac{y}{r}+\cos \frac{t_{i}}{r} \xi_{y} .
$$

This vector is normal to $V_{i}$ just like $\xi_{x}^{\prime}$ in (5); indeed, $\xi_{x}^{\prime}=g_{i}(x)$.

We compute the differential of $g_{i}$ at $x$. For $X \in T_{i}(x)$ we have

$$
\begin{aligned}
\left(g_{i}\right)_{*}(X) & =-\sin \frac{t_{i}}{r} \frac{X}{r}+\cos \frac{t_{i}}{r} D_{X} \xi=-\sin \frac{t_{i}}{r} \frac{X}{r}+\cos \frac{t_{i}}{r}(-A X) \\
& =-\sin \frac{t_{i}}{r} \frac{X}{r}-\cos \frac{t_{i}}{r} \cot \frac{t_{i}}{r} \frac{X}{r}=-\frac{1}{\sin \left(t_{i} / r\right)} \frac{X}{r}
\end{aligned}
$$

(9) shows that $\left(g_{i}\right)_{*}$ is injective.

We consider the linear function $w$ on the normal space $N_{p}\left(v_{i}\right)$ given by

$$
w(Z)=\operatorname{trace} B_{Z} .
$$

We shall show that $w$ is identically 0 ; then (6) shows that the constant

$$
q=\sum_{j \neq i} \nu_{j} \frac{-c+a_{i} a_{j}}{a_{i}-a_{j}}
$$

is equal to 0 .

Let $H_{p}$ be the connected component containing $\xi_{x}^{\prime}$ of the set of all timelike unit vectors in $N_{p}\left(V_{i}\right)$. Then $g_{i}\left(M_{i}\right) \subset H_{p}$. Since $\left(g_{i}\right)_{*}$ is injective, we 
see that $g_{i}\left(M_{i}\right)$ is an open subset of $H_{p}$. Since (6) is valid for every point of $M_{i}$, it follows that $w(Z)=q$ for all $Z \in H_{p}$. On the other hand, (7) shows that $w(Z)=0$ for all $Z \in T_{i}(x)$, considered as a subspace of $N_{p}\left(V_{i}\right)$.

Our assertion $w \equiv 0$ on $N_{p}\left(V_{i}\right)$ will follow from the following lemma which is easily proved.

Lemma. Let $L^{m+1}$ be the Lorentzian vector space and let

$$
H^{m}=\left\{x=\left(x_{1}, x_{2}, \cdots, x_{m+1}\right) ;\langle x, x\rangle=-1, x_{1}>0\right\} .
$$

Suppose $w$ is a linear function on $L^{n+1}$ such that

(1) $w \equiv q$, constant, on $H^{m}$

(2) $w(e)=0$ for some space-like unit vector.

Then $w \equiv 0$ on $L^{m+1}$.

\section{§4. Examples}

It is easy to determine all complete space-like umbilical hypersurfaces in $L^{n+1}, S_{1}^{n+1}(c), c>0$, and $H_{1}^{n+1}(c), c<0$.

We shall give a list of all complete space-like hypersurfaces with two distinct constant principal curvatures in each of the model space forms $(c=$ $0,1,-1)$. The shape operator is parallel.

1) In $L^{n+1}$, they are

$$
H^{k}\left(-\frac{1}{r^{2}}\right) \times E^{n-k}=\left\{\left(x_{1}, x_{2}, \cdots, x_{n+1}\right) ;-x_{1}^{2}+x_{2}^{2}+\cdots x_{k+1}^{2}=-r^{2}\right\}
$$

where $0<k<n$.

For the time-like unit normal

$$
\xi=-\left(\frac{x_{1}}{r}, \cdots, \frac{x_{k+1}}{r}, 0, \cdots, 0\right)
$$

the principal curvatures are

$$
\frac{1}{r} \text { and } 0
$$

with multiplicities $k$ and $n-k$, respectively.

2) In $S_{1}^{n+1}(1)$, they are

$$
\begin{array}{r}
H^{k}\left(-\frac{1}{r^{2}}\right) \times S^{n-k}\left(\frac{1}{1+r^{2}}\right)=\left\{\left(x_{1}, \cdots, x_{k+1}, \cdots, x_{n+2}\right)\right. \\
\left.-x_{1}^{2}+\sum_{i=2}^{k+1} x_{i}^{2}=-r^{2} \text { and thus } \sum_{j=k+2}^{n+2} x_{j}^{2}=1+r^{2}\right\}
\end{array}
$$


where

$$
r>0
$$

and

$$
0<k<n \text {. }
$$

For the time-like unit normal

$$
\xi=-\left(\frac{\sqrt{1+r^{2}}}{r} x_{1}, \cdots, \frac{\sqrt{1+r^{2}}}{r} x_{k}, \frac{r}{\sqrt{1+r^{2}}} x_{k+1}, \cdots, \frac{r}{\sqrt{1+r^{2}}} x_{n+2}\right)
$$

the principal curvatures are

$$
\frac{\sqrt{1+r^{2}}}{r} \text { and } \frac{r}{\sqrt{1+r^{2}}}
$$

of multiplicities $k$ and $n-k$, respectively.

3) In $H_{1}^{n+1}(-1)$, they are

$$
\begin{gathered}
H^{k}\left(-\frac{1}{r^{2}}\right) \times H^{n-k}\left(\frac{1}{r^{2}-1}\right)=\left\{\left(x_{1}, x_{2}, x_{3}, \cdots, x_{k+2}, x_{k+3}, \cdots, x_{n+2}\right) ;\right. \\
\left.-x_{1}^{2}+\sum_{i=3}^{k+2} x_{i}^{2}=-r^{2} \text { and thus }-x_{2}^{2}+\sum_{j=k+3}^{n+2} x_{j}^{2}=r^{2}-1\right\}
\end{gathered}
$$

where

$$
0<k<n, \quad 0<r<1 .
$$

For the time-like unit normal

$$
\begin{gathered}
\xi=-\left(\frac{\sqrt{1-r^{2}}}{r} x_{1}, \frac{-r}{\sqrt{1-r^{2}}} x_{2}, \frac{\sqrt{1-r^{2}}}{r} x_{3}, \cdots, \frac{\sqrt{1-r^{2}}}{r} x_{k+2},\right. \\
\left.\frac{-r}{\sqrt{1-r^{2}}} x_{k+3}, \cdots, \frac{-r}{\sqrt{1-r^{2}}} x_{n+2}\right)
\end{gathered}
$$

the principal curvatures are

$$
\frac{\sqrt{1-r^{2}}}{r} \text { and } \frac{-r}{\sqrt{1-r^{2}}}
$$

of multiplicities $k$ and $n-k$. 


\section{References}

[1] K. Nomizu, Some results in E. Cartan's theory of isoparametric families of hypersurfaces, Bull. Amer. Math. Soc., 79 (1973), 1184-1188.

[2] K. Nomizu, Elie Cartan's work on isoparametric families of hypersurfaces, Proc. Symposia in Pure Math., 27 (1975), 191-200, Amer. Math. Soc..

The work of H. F. Münzner mentioned in [2] has now appeared: Isoparametrische Hyperflächerı in Sphären, Math. Ann., 251 (1980), 57-71.

Department of Mathematics

BROWN UNIVERSITY

Providence, R.I.

02912 